\title{
DIFFERENTIABILITY AND LOCAL ROTUNDITY
}

\author{
A. C. YORKE
}

(Received 5 July 1978)

Communicated by E. Strzelecki

\begin{abstract}
The concept of very weak local uniform rotundity (very WLUR) is introduced. It is shown that if a Banach space $E$ is WLUR, very WLUR, or LUR then its dual $E^{*}$ is smooth, very smooth, or Fréchet differentiable, respectively, on a norm dense subset. This leads to examples of nonreflexive spaces which are Fréchet differentiable at every nonzero point of a (relatively) norm dense subset of the embedding of $E^{* *}$ in the fourth dual. When $E$ is reflexive, necessary and sufficient conditions for $E$ and $E^{*}$ to be WLUR and LUR are given.
\end{abstract}

Subject classification (Amer. Math. Soc. (MOS) 1970): 46 B 99.

\section{Introduction}

The main purpose of this paper is to investigate the differentiability of the norm of a Banach space $E$ at nonzero points of $\hat{E}^{*}$ and $\hat{E}^{* *}$, the embeddings of $E^{*}$ and $E^{* *}$ in the third and fourth duals, respectively. It is well known that if the third dual of $E$ is smooth (Gâteaux differentiable) at every nonzero point of $\hat{E}^{*}$, then $E$ must be reflexive. One of the results given here is that this cannot be weakened, even in the fourth dual. It is shown that a space can be smooth, in fact Fréchet differentiable, at every nonzero point of a (relatively) norm dense subset of $E^{* *}$ and still not be reflexive. If this norm dense subset is uniformly Gâteaux differentiable, however, the space must be reflexive.

Firstly, local rotundity at points of $E$ is related to the differentiability of the norm at points of $E^{*}$. This is done by using the equivalent definitions of WLUR and LUR which are given in Lemma 2 of Section 3. These definitions show that there is a local rotundity condition which lies between WLUR and LUR: this concept is called very weak local uniform rotundity (very WLUR) here. It is shown that very WLUR at points of $E$ gives information about very smoothness at points of $E^{*}$, or equivalently, smoothness at points of $\hat{E}^{*}$. 
Next the problem of trying to reverse these implications is considered. Lovaglia (1955) showed that, even in reflexive spaces, differentiability of the norm cannot be used to obtain information about local rotundity, unless an additional condition is added. The condition added here is similar to Lovaglia's. Section 4 looks at this problem and applies the results to reflexive spaces. The results given here recover those of Lovaglia (1955), and extend these results to WLUR spaces.

\section{Definitions and notation}

Let $E$ be a real Banach space, $E^{(n)}$ its $n$th dual $\left(E^{(0)}=E\right)$, and $S\left(E^{(n)}\right)$ the unit sphere of $E^{(n)}$. The spaces $E^{(1)}$ and $E^{(2)}$ will be denoted by $E^{*}$ and $E^{* *}$, respectively. For each $n$ let $Q_{n}$ denote the mapping which embeds $E^{(n)}$ in $E^{(n+2)}$. The spaces $Q_{0} E, Q_{1} E^{*}, Q_{2} E^{* *}, Q_{2} Q_{0} E$ and $Q_{3} Q_{1} E^{*}$ will be denoted by $\hat{E}, \hat{E}^{*}, \hat{E}^{* *}, \hat{E}$ and $\hat{E}^{*}$, respectively.

Dixmier (1948) showed that the third dual may be represented as $\hat{E}^{*}+E^{\perp}$, with $E^{\perp}=\operatorname{ker} Q_{0}^{*}$, while the fourth dual has two distinct representations: $\hat{E}^{* *}+E^{* \perp}$ and $E^{\perp \perp}+E^{* \perp}$. Here $E^{* \perp}=\operatorname{ker} Q_{1}^{*}$ and $E^{\perp \perp}$ is the range of the linear isometry $Q_{0}^{* *}: E^{* *} \rightarrow E^{(4)}$.

The set-valued mapping $D_{E}: E \rightarrow 2^{E^{*}}$ which assigns to each $x \in E$ the

$$
\left\{f \in E^{*}: f(x)=\|f\| \quad\|x\| \text { and }\|f\|=\|x\|\right\}
$$

is called the duality mapping. The duality mappings on $E^{(n)}$, for $n>0$, will be denoted by $D_{n}$. The set $D(S(E))$ will be denoted by $D(S)$; similarly $D_{n}\left(S\left(E^{(n)}\right)\right.$ will be denoted by $D_{n}(S)$. Elements of $D(x)$ will be represented as $f_{x}$.

The mapping $x \rightarrow f_{x}$ which associates each $x \in S(E)$ with one $f_{x} \in D(x)$, and has the property that $f_{\lambda x}=\lambda f_{x}$ for all real $\lambda>0$, is called a support mapping. A support mapping is said to be norm to $T$ continuous at $x \in S(E)$ if it is continuous at $x$ when $E$ has the norm topology and $E^{*}$ the $T$ topology.

$E$ is smooth at $x \in S(E)$ if the norm of $E$ is Gâteaux differentiable ( $G$-differentiable) at $x$; very smooth at $x$ if the norm of $E^{* *}$ is $G$-differentiable at $\hat{x}$; Fréchet differentiable (F-differentiable) at $x$ if the norm of $E$ is $F$-differentiable at $x$. Equivalently, $E$ is smooth, very smooth, or $F$-differentiable at $x$ if and only if every support mapping on $E$ is norm to $\sigma\left(E^{*}, E\right)$, norm to $\sigma\left(E^{*}, E^{* *}\right)$, or norm to norm continuous at $x$, respectively. (See Giles (1975.) $E$ is said to be smooth, very smooth or F-differentiable if it has that property for all $x \in S(E)$. $E$ is extremely smooth at $F \in S\left(E^{* *}\right)$ if the norm of $E^{* *}$ is $G$-differentiable at $F$ in the $\hat{x}$ direction for all $\hat{x} \in S(\hat{E})$. This is equivalent to saying that if $\mathscr{F}, \mathscr{G} \in D_{2}(F)$, then $\mathscr{F}-\mathscr{G} \in E^{\perp} . E$ is extremely smooth if it is extremely smooth at $F$ for every $F \in S\left(E^{* *}\right)$ (Sullivan (1975)).

$E$ is rotund if $x, y \in S(E)$ and $\|x+y\|=2$ implies $x=y . E$ is weakly locally 
uniformly rotund (WLUR) at $x \in S(E)$ in the $f \in S\left(E^{*}\right)$ direction if every sequence (or net) $\left\{x_{n}\right\}$ in $S(E)$ with $\left\|x_{n}+x\right\| \rightarrow 2$ has $f\left(x_{n}-x\right) \rightarrow 0$. E is WLUR at $x$ if $E$ is WLUR at $x$ in the $f$ direction for every $f \in S\left(E^{*}\right) . E$ is locally uniform rotund $(L U R)$ at $x \in S(E)$ if every sequence (or net) $\left\{x_{n}\right\}$ in $S(E)$ with $\left\|x_{n}+x\right\| \rightarrow 2$ has $\left\|x_{n}-x\right\| \rightarrow 0$. $E$ is WLUR (LUR) if it is WLUR (LUR) at $x$ for every $x \in S(E)$.

$E^{*}$ is weak -* locally uniformly rotund $\left(W^{*} L U R\right)$ at $f \in S\left(E^{*}\right)$ if $E^{*}$ is WLUR at $f$ in the $\hat{x}$ direction for every $\hat{x} \in S(\hat{E}) . E^{*}$ is $W^{*} L U R$ if it is $W^{*} L U R$ at $f$ for every $f \in S\left(E^{*}\right)$.

\section{Local rotundity}

This section uses local rotundity methods to investigate the differentiability of the norm at certain points of $S(E), S\left(E^{*}\right)$ and $S\left(E^{* *}\right)$. In order to do this new definitions of WLUR and LUR must be given. Firstly, however, an easy lemma is needed.

LEMMA 1. Let $x \in S(E)$ and $x \rightarrow f_{x}$ be any support mapping on $E$. If $\left\{x_{n}\right\}$ is a sequence (or net) in $S(E)$ with $\left\|x_{n}-x\right\| \rightarrow 0$, then $\left\|f_{x_{n}}+f_{x}\right\| \rightarrow 2$.

Proof. This follows from the equation

$$
2\left(\|x\|^{2}+\|y\|^{2}\right)=\left(f_{x}+f_{y}\right)(x+y)+\left(f_{x}-f_{y}\right)(x-y) .
$$

LEMMA 2. Let $x \in S(E)$.

(1) $E$ is WLUR at $x$ in the $f \in S\left(E^{*}\right)$ direction if and only if every sequence (or net) $\left\{F_{n}\right\}$ in $S\left(E^{* *}\right)$ with $\left\|F_{n}+\hat{x}\right\| \rightarrow 2$ has $\left(F_{n}-\hat{x}\right)(f) \rightarrow 0$.

(2) $E$ is LUR at $x$ if and only if every sequence (or net) $\left\{F_{n}\right\}$ in $S\left(E^{* *}\right)$ with $\left\|F_{n}+\hat{x}\right\| \rightarrow 2$ has $\left\|F_{n}-\hat{x}\right\| \rightarrow 0$.

Proof. (1) Clearly if the condition holds, $E$ is WLUR at $x$ in the $f$ direction. Therefore, assume there is a sequence $\left\{F_{n}\right\}$ in $S\left(E^{* *}\right)$ and an $\varepsilon>0$ such that $\left\|F_{n}+\hat{x}\right\| \rightarrow 2$, but $\left(F_{n}-\hat{x}\right)(f) \geqslant \varepsilon$ for all $n$. For each (fixed) $n$, let $\left\{g_{n, m}\right\}$ be a norming sequence for $F_{n}+\hat{x}$; that is, $g_{n, m} \in S\left(E^{*}\right)$ for each $m$ and

$$
\left(F_{n}+\hat{x}\right)\left(g_{n, m}\right)>\left\|F_{n}+\hat{x}\right\|-1 / m, \quad m=1,2,3, \ldots .
$$

Let $\left\{f_{n}\right\}=\left\{g_{n, n}\right\}$ be the diagonal sequence obtained from $\left\{g_{n, m}\right\}$, and $V_{n}$ denote the $\sigma\left(E^{* *}, E^{*}\right)$ neighbourhood of $F_{n}$ determined by $f_{n}, f$ and $1 / n$. Since $S(\hat{E})$ is $\sigma\left(E^{* *}, E^{*}\right)$ dense in $S\left(E^{* *}\right)$, there is an $\hat{x}_{n} \in V_{n},\left\|\hat{x}_{n}\right\|=1$, for each $n$. This gives a sequence $\left\{\hat{x}_{n}\right\}$ in $S(E)$ with the following properties: and

(i) $2 \geqslant\left\|\hat{x}_{n}+\hat{x}\right\| \geqslant\left(x_{n}+\hat{x}\right)\left(f_{n}\right)>\left(F_{n}+\hat{x}\right)\left(f_{n}\right)-1 / n>\left\|F_{n}+\hat{x}\right\|-2 / n$,

(ii) $0<\varepsilon \leqslant\left(F_{n}-\hat{x}\right)(f)<\left(\hat{x}_{n}-\hat{x}\right)(f)+1 / n$. 
Thus, although $\left\|x_{n}+x\right\| \rightarrow 2$ as $n \rightarrow \infty$, the sequence $\left\{f\left(x_{n}-x\right)\right\}$ remains bounded away from zero for all $n$. Hence $E$ cannot be WLUR at $x$ in the $f$ direction. The proof for nets is similar.

(2) Once again it is clear that if the condition holds, $E$ is LUR at $x$. Conversely, if the condition does not hold there is a sequence $\left\{F_{n}\right\}$ in $S\left(E^{* *}\right)$ and an $\varepsilon>0$ such that $\left\|F_{n}+\hat{x}\right\| \rightarrow 2$, but $\left\|F_{n}-\hat{x}\right\| \geqslant \varepsilon$ for all $n$. For each $n$ let $\left.\} g_{n, m}\right\}$ and $\left\{k_{n, m}\right\}$ be norming sequences for $\left\|F_{n}+\hat{x}\right\|$ and $\left\|F_{n}-\hat{x}\right\|$, respectively. Denote the diagonal sequences $\left\{g_{n, n}\right\}$ and $\left\{k_{n, n}\right\}$ by $\left.\} g_{n}\right\}$ and $\left\{k_{n}\right\}$, respectively, and let $V_{n}$ denote the $\sigma\left(E^{* *}, E^{*}\right)$ neighbourhood of $F_{n}$ determined by $g_{n}, k_{n}$ and $1 / n$. Proceeding as in part (1) gives a sequence $\left\{\hat{x}_{n}\right\}$ in $S(\hat{E})$ such that:

(i) $2 \geqslant\left\|\hat{x}_{n}+\hat{x}\right\| \geqslant\left(\hat{x}_{n}+\hat{x}\right)\left(g_{n}\right)>\left(F_{n}+\hat{x}\right)\left(g_{n}\right)-1 / n>\left\|F_{n}+\hat{x}\right\|-2 / n$, and

(ii) $0<\varepsilon \leqslant\left\|F_{n}-\hat{x}\right\|<\left(F_{n}-\hat{x}\right)\left(k_{n}\right)+1 / n<\left(\hat{x}_{n}-\hat{x}\right)\left(k_{n}\right)+2 / n \leqslant\left\|x_{n}-x\right\|+2 / n$.

Thus the sequence $\left\{\left\|x_{n}-x\right\|\right\}$ cannot converge to zero, even though $\left\|x_{n}+x\right\| \rightarrow 2$ as $n \rightarrow \infty$. Hence $E$ is not LUR at $x$. As in part (1), the proof using nets is similar, so will be omitted.

This lemma motivates the following definition: $E$ is very weakly locally uniformly rotund (very WLUR) at $x \in S(E)$ in the $\mathscr{F} \in S\left(E^{(3)}\right)$ direction if every sequence (or net) $\left\{F_{n}\right\}$ in $S\left(E^{* *}\right)$ with $\left\|F_{n}+\hat{x}\right\| \rightarrow 2$ has $\mathscr{F}\left(F_{n}-\hat{x}\right) \rightarrow 0 . E$ is very WLUR at $x$ if it is very WLUR at $x$ in the $\mathscr{F}$ direction for every $\mathscr{F} \in S\left(E^{(3)}\right) . E$ is very WLUR if it is very WLUR at $x$ for every $x \in S(E)$.

The next lemma is an immediate consequence of Lemma $2:$ it will be needed in the proof of Theorem 2(3).

LEMMA 3. Let $x \in S(E) . E$ is very WLUR at $x$ in the $\mathscr{F} \in S\left(E^{(3)}\right)$ direction if and only if every sequence (or net) $\left\{x_{n}^{(4)}\right\}$ in $S\left(E^{(4)}\right)$ with $\left\|x_{n}^{(4)}+\hat{x}\right\| \rightarrow 2$ has $\left(x_{n}^{(4)}-\hat{x}\right)(\mathscr{F}) \rightarrow 0$.

Therefore, $E$ is very WLUR at $x$ if and only if $\hat{E}$ is $W^{*} L U R$ at $\hat{x}$.

THEOREM 1. Let $x \in S(E)$.

(1) If $E$ is WLUR at $x, E^{*}$ is smooth at each $f_{x} \in D(x)$.

(2) If $E$ is very WLUR at $x, E^{*}$ is very smooth at each $f_{x} \in D(x)$.

(3) If $E$ is LUR at $x, E^{*}$ is F-differentiable at each $f_{x} \in D(x)$.

Proof. (1) Assume $E^{*}$ is not smooth at one of the $f_{x}$ in $D(x)$. Then there is a sequence $\left\{f_{n}\right\}$ in $S\left(E^{*}\right)$, a $g$ in $S\left(E^{*}\right)$, and an $\varepsilon>0$ such that $\left\|f_{n}-f_{x}\right\| \rightarrow 0$, but $\left|\left(F_{f_{n}}-\hat{x}\right)(g)\right| \geqslant \varepsilon$ for all $n$. By Lemma $1,\left\|f_{n}-f_{x}\right\| \rightarrow 0$ implies $\left\|F_{f_{n}}+\hat{x}\right\| \rightarrow 2$. But the sequence $\left\{\left(F_{f_{n}}-\hat{x}\right)(g)\right\}$ is bounded away from zero for all $n$; so, by Lemma 2(1), $E$ cannot be WLUR at $x$ in the $g$ direction.

(2) and (3) are proved similarly. 
Therefore:

(1) If $E$ is WLUR, $E^{*}$ is smooth at each $f \in D(S)$.

(2) If $E$ is very WLUR, $E^{*}$ is very smooth at each $f \in D(S)$.

(3) If $E$ is LUR, $E^{*}$ is $F$-differentiable at each $f \in D(S)$.

There is another type of local rotundity to be considered when dealing with dual spaces, namely W*LUR. Cudia (1963) has shown that W*LUR at points of $E^{*}$ gives information about smoothness at points of $E$. The following lemma shows that $\mathrm{W}^{*} \mathrm{LUR}$ in $E^{*}$ actually gives information about smoothness at points of $E^{* *}$.

LEMma 4. Let $f \in S\left(E^{*}\right)$. It $E^{*}$ is $W^{*} L U R$ at $f$, then $E$ is extremely smooth at each $F_{f} \in D_{1}(f)$.

Proof. If $E$ is not extremely smooth at $F_{f} \in D_{1}(f)$ there is an $\mathscr{F} \in D_{2}\left(F_{f}\right)$, a $\hat{y} \in S(\hat{E})$, and an $\varepsilon>0$ such that $\mid(\mathscr{F}+\hat{f}))(\hat{y}) \mid \geqslant \varepsilon$. Let $V_{n}$ denote the $\left(E^{(3)}, E^{* *}\right)$ neighbourhood of $\mathscr{F}$ determined by $F_{f}, \hat{y}$ and $1 / n$. The usual 'weak-* density' argument gives a sequence $\left\{\hat{f}_{n}\right\}$ in $S\left(\hat{E}^{*}\right)$ such that

and

$$
2 \geqslant\left\|f_{n}+f\right\| \geqslant\left(\hat{f}_{n}+\hat{f}\right)\left(F_{f}\right)>(\mathscr{F}+\hat{f})\left(F_{f}\right)-1 / n=2-1 / n,
$$

$$
0<\varepsilon \leqslant|(\mathscr{F}-\hat{f})(\hat{y})|<\left|\left(f_{n}-f\right)(y)\right|+1 / n .
$$

Thus $E^{*}$ cannot be $\mathrm{W}^{*} \mathrm{LUR}$ at $f$. Since $F_{f} \in D_{1}(f)$ was chosen arbitrarily, the result follows.

Now apply Theorem 1 to $E^{*}$. By using Lemma 4 , and the fact that $\hat{x}$ is always in $D_{1}\left(f_{x}\right)$, one gets the following result.

CoROLlaRY 1. Let $f_{x} \in D(x)$.

(1) If $E^{*}$ is $W^{*} L U R$ at $f_{x}, E$ is smooth at $x$.

(2) If $E^{*}$ is WLUR at $f_{x}, E$ is very smooth at $x$.

(3) If $E^{*}$ is very WLUR at $f_{x}$, $\hat{E}$ is smooth at $\hat{x}$.

(4) If $E^{*}$ is LUR at $x, E$ is F-differentiable at $x$.

An immediate consequence of Corollary 1 is that if $E^{*}$ is $W^{*}$ LUR, WLUR or LUR at every $f \in D(S)$, then $E$ is smooth, very smooth or $F$-differentiable, respectively. This gives a (slight) improvement to the results given in Lovaglia (1955), Cudia (1963) and Diestel and Faires (1974).

Theorem 1(2) shows that if $E$ is very WLUR then $Q_{1}(D(S))$ is smooth in $E^{(3)}$. However, combining Theorem 1(3) with Proposition 7 of Yorke (1977) gives a stronger result: if $E$ is LUR then $S\left(E^{(3)}\right)$ is $F$-differentiable at each $\hat{f} \in Q_{1}(D(S))$. Thus smoothness, or even $F$-differentiability, on this (relatively) norm dense subset of $S\left(E^{*}\right)$ is not sufficient to imply reflexivity.

The usual situation in Banach space is that if a geometric condition gives a certain property on a space, a weaker condition will give the same (or stronger) 
property on the dual space. This is not the case, however, when considering differentiability in $\hat{E}^{*}$ and $\hat{E}^{* *}$. Zizler (1969) has shown that any separable dual space can be renormed with a LUR dual norm. Applying Theorem 1(3) and Proposition 7 of Yorke (1977) to a nonreflexive space with a separable dual shows that this space admits an equivalent norm with the property that $S\left(E^{(4)}\right)$ is $F$-differentiable at every point of $Q_{2}\left(D_{1}(S)\right)$. Thus local differentiability conditions on $Q_{1}(D(S))$ and $Q_{2}\left(D_{1}(S)\right)$ are too weak to give reflexivity. This leads to considering $U G$-differentiability on these sets.

To say that $Q_{1}(D(S))$ is $U G$-differentiable means that every support mapping on $E^{(3)}$ is uniformly continuous norm to $\sigma\left(E^{(4)}, E^{(3)}\right)$ when restricted to $Q_{1}(D(S)$ ).

LEMMA 5. If $Q_{1}(D(S))$ is UG-differentiable, then $E$ is reflexive.

Proof. Choose any support mapping on $E^{(3)}$ for which $\hat{f} \rightarrow \hat{F}_{f}$; this gives a support mapping $f \rightarrow \hat{F}_{f}$ on $E^{*}$. Let $\left\{f_{n}\right\}$ and $\left\{g_{n}\right\}$ be any two sequences in $S\left(E^{*}\right)$ such that $\left\|f_{n}-g_{n}\right\| \rightarrow 0$ as $n \rightarrow \infty$. Since $D(S)$ is norm dense in $S\left(E^{*}\right)$ there are sequences $\left\{f_{x_{n}}\right\}$ and $\left\{g_{y_{n}}\right\}$ in $D(S)$ such that $\left\|f_{n}-f_{x_{n}}\right\| \rightarrow 0$ and $\left\|g_{n}-g_{y_{n}}\right\| \rightarrow 0$. Now since $Q_{1}(D(S))$ is $U G$-differentiable, $\left\|f_{n}-f_{x_{n}}\right\|=\left\|\hat{f}_{n}-\hat{f}_{x_{n}}\right\| \rightarrow 0$ implies $\left(\hat{F}_{f_{n}}-\hat{x}_{n}\right)(\mathscr{G}) \rightarrow 0$ for every $\mathscr{G} \in S\left(E^{(3)}\right)$. Similarly $\left|\left(\hat{G}_{g_{n}}-\hat{y}_{n}\right)(\mathscr{G})\right| \rightarrow 0$. But

$$
\left|\left(\hat{G}_{g_{n}}-\hat{F}_{f_{n}}\right)(\mathscr{G})\right| \leqslant\left|\left(\hat{G}_{g_{n}}-\hat{y}_{n}\right)(\mathscr{G})\right|+\left|\left(\hat{y}_{n}-\hat{x}_{n}\right)(\mathscr{G})\right|+\left|\left(\hat{x}_{n}-\hat{F}_{f_{n}}\right)(\mathscr{G})\right|,
$$

and as all three terms on the right converge to zero, $\mathscr{G}\left(G_{g_{n}}-F_{f_{n}}\right) \rightarrow 0$ as well. Thus the support mapping $f \rightarrow F_{f}$ is uniformly continuous norm to $\sigma\left(E^{*}, E^{* *}\right)$ on $E^{*}$. Hence $E^{*}$ is very smooth (Giles (1975)), or equivalently, $\hat{E}^{*}$ is smooth, so $E$ must be reflexive.

\section{Differentiability}

This section considers the problem of reversing the implications of Theorem 1. As in Lovaglia (1955) this cannot be done, even in reflexive spaces, unless some sort of local rotundity condition is added.

THEOREM 2. Let $E^{*}$ be WLUR at $f \in S\left(E^{*}\right)$ in the $F_{f} \in D_{1}(f)$ direction.

(1) If $E$ is extremely smooth at $F_{f}, E^{*}$ is $W^{*} L U R$ at $f$.

(2) If $E^{* *}$ is smooth at $F_{f}, E^{*}$ is WLUR at $f$.

(3) If $E^{* *}$ is very smooth at $F_{f}, E^{*}$ is very WLUR at $f$.

(4) If $E^{* *}$ is F-differentiable at $F_{f}, E^{*}$ is LUR at $f$.

Proof. (1) If $E^{*}$ is not $\mathrm{W}^{*} \mathrm{LUR}$ at $f$, there is a sequence (or net) $\left\{f_{n}\right\}$ in $S\left(E^{*}\right)$, a $y \in S(E)$, and an $\varepsilon>0$ such that $\left\|f_{n}+f\right\| \rightarrow 2$, but $\left|\left(f_{n}-f\right)(y)\right| \geqslant \varepsilon$ for all $n$. Let $\mathscr{F}$ be a $\sigma\left(E^{(3)}, E^{* *}\right)$ cluster point of the $\left\{\hat{f}_{n}\right\}$. Since the norm is $\sigma\left(E^{(3)}, E^{* *}\right)$ lower 
semi-continuous, $\|\mathscr{F}\| \leqslant 1$. But $E^{*}$ is WLUR at $f$ in the $F_{f}$ direction. So $\left\|f_{n}+f\right\| \rightarrow 2$ implies $\hat{f}_{n}\left(F_{f}\right) \rightarrow 1$ and hence $\mathscr{F} \in D_{2}\left(F_{f}\right)$. However $\mathscr{F}(\hat{y}) \neq \hat{f}(\hat{y})$, so $E$ cannot be extremely smooth at $F_{f}$.

(2) If $E^{*}$ is not WLUR at $f$, there is a sequence (or net) $\left\{\mathscr{F}_{n}\right\}$ in $S\left(E^{(3)}\right)$, an $F \neq F_{f} \in S\left(E^{* *}\right)$, and an $\varepsilon>0$ such that $\left\|F_{n}+f\right\| \rightarrow 2$ but $\left|\left(\mathscr{F}_{n}-\hat{f}\right)(F)\right| \geqslant \varepsilon$ for all $n$. Once again, since $E^{*}$ is WLUR in the $F_{f}$ direction, $\mathscr{F}_{n}\left(F_{f}\right) \rightarrow 1$. Now applying the Bishop-Phelps-Bollobás Theorem (Bollobás (1972)) gives a sequence (or net) $\left\{F_{n}\right\}$ in $S\left(E^{* *}\right)$ such that $\left\|F_{n}-F_{f}\right\| \rightarrow 0$ and $\left\|\mathscr{F}_{F n}-\mathscr{F}_{n}\right\| \rightarrow 0$ as $n \rightarrow \infty$. However, since

$$
0<\varepsilon \leqslant\left|\left(\mathscr{F}_{n}-\hat{f}\right)(F)\right| \leqslant\left\|\mathscr{F}_{n}-\mathscr{F}_{F n}\right\|+\left|\left(\mathscr{F}_{F n}-\hat{f}\right)(F)\right|,
$$

the sequence (or net) $\left\{\left(\mathscr{F}_{F_{n}}-\hat{f}\right)(F)\right\}$ cannot converge to zero. Thus any support mapping on $E^{* *}$ for which $F_{n} \rightarrow \mathscr{F}_{F_{n}}$ and $F_{f} \rightarrow \hat{f}$ fails to be norm to $\sigma\left(E^{(3)}, E^{* *}\right)$ continuous at $F_{f}$, so $E^{* *}$ is not smooth at $F_{f}$ (Giles (1971)).

(3) and (4) are proved similarly. The proof of (3) uses Lemma 3.

Corollary 2. Let $f_{x} \in D(S)$, and assume $E^{*}$ is WLUR at $f_{x}$ in the $\hat{x}$ direction.

(1) If $E$ is smooth at $x, E^{*}$ is $W^{*} L U R$ at $f_{x}$.

(2) If $E$ is very smooth at $x, E^{*}$ is WLUR at $f_{x}$.

(3) If $E$ is smooth at $\hat{x}, E^{*}$ is very WLUR at $f_{x}$.

(4) If $E$ is F-differentiable at $x, E^{*}$ is LUR at $f_{x^{*}}$

This follows immediately from Theorem 2 since $E$ is smooth at $x$ if and only if $E$ is extremely smooth at $\hat{x}, \hat{E}$ is very smooth at $\hat{x}$ if and only if $E^{* *}$ is smooth at $\hat{x}$, $E$ is smooth at $x$ if and only if $E^{* *}$ is very smooth at $\hat{x}$, and $E$ is $F$-differentiable at $x$ if and only if $E^{* *}$ is $F$-differentiable at $\hat{x}$ (Yorke (1977), Proposition 7).

Now apply Theorem 2 to $E^{*}$, and use the fact that $\hat{x} \in D_{1}\left(f_{x}\right)$.

COROLlary 3. Let $x \in S(E)$, and assume that $E$ is WLUR at $x$ in the $f_{x}$ direction, for at least one $f_{x} \in D(x)$.

(1) If $E^{*}$ is smooth at $f_{x}, E$ is WLUR at $x$.

(2) If $E^{*}$ is very smooth at $f_{x}, E$ is very WLUR at $x$.

(3) If $E^{*}$ is F-differentiable at $f_{x}, E$ is LUR at $x$.

According to Lovaglia (1955), page $241, E$ is weakly locally uniformly convex (weakly l.u.c.) at $x \in S(E)$ if every sequence $\left\{x_{n}\right\}$ in $S\left(E^{*}\right)$ with $\left\|x_{n}+x\right\| \rightarrow 2$ has $f_{x}\left(x_{n}-x\right) \rightarrow 0$ for each $f_{x} \in D(x)$. This expression is not used here. Notice that the conditions given in Theorem 2, Corollary 2, and Corollary 3 are somewhat weaker than Lovaglia's weak local uniform convexity.

COROLLARY 4. Let $E$ be a reflexive space. If $E$ is WLUR at $x$ in the $f_{x}$ direction, for at least one $f_{x} \in D(x)$, then

(1) $E$ is WLUR if and only if $E^{*}$ is smooth;

(2) $E$ is LUR if and only if $E^{*}$ is F-differentiable. 
Proof. Since $E$ is reflexive, $D(S)=S\left(E^{*}\right)$ (James (1964)), so (1) follows from Theorem 1(1) and Corollary 3(1), while (2) is a consequence of Theorem 1(3) and Corollary 3(3).

CoROLLARY 5. Let $E$ be a reflexive space. If $E^{*}$ is WLUR at each $f \in S\left(E^{*}\right)$ in the $x$ direction, for at least one $x \in D_{1}(f)$, then

(1) $E^{*}$ is WLUR if and only if $E$ is smooth;

(2) $E^{*}$ is LUR if and only if $E$ is F-differentiable.

PROof. (1) follows from Corollary 2(1) and Corollary 1(1). (2) follows from Corollary 2(4) and Corollary 1(4).

Part (2) of Corollary 4 is essentially Theorem 2.5 of Lovaglia (1955), while Corollary 5(2) recovers Theorem 2.7 of Lovaglia (1955).

Since smoothness and rotundity are dual concepts in reflexive spaces, Corollary 4(1) shows that a reflexive space is WLUR if and only if it is rotund and WLUR at each $x \in S(E)$ in the $f_{x}$ direction, for at least one $f_{x} \in D(x)$. The following example shows that this extra condition cannot be removed.

EXAmple. (Smith (1978).) Consider $l_{2}$ with its usual norm $\|\cdot\|_{2}$. For each $x=\left(x_{1}, x_{2}, x_{3}, \ldots\right)$ in $l_{2}$ let $\tilde{x}=\left(0, x_{2}, x_{3}, \ldots\right)$, and define $\|x\|_{0}=\left|x_{1}\right|+\|\tilde{x}\|_{2}$. This is an equivalent norm for $l_{2}$. If $\left\{\alpha_{n}\right\}$ is a sequence of positive real numbers with $\alpha_{n} \rightarrow 0$, define $T: l^{2} \rightarrow l^{2}$ by $T\left(x_{1}, x_{2}, x_{3}, \ldots\right)=\left(\alpha_{2} x_{2}, \alpha_{3} x_{3}, \ldots\right)$, and let

$$
\text { | }\|x\| \|=\left(\|x\|_{0}^{2}+\left\|T_{x}\right\|_{2}^{2}\right)^{\frac{1}{2}} .
$$

This is also an equivalent norm for $l_{2}$.

Let $E=\left(l_{2},\||\cdot|\|\right)$. A straightforward calculation shows that the norm and $\sigma\left(E, E^{*}\right)$ topologies agree on $S(E)$, and that $E$ is rotund. Thus, since $E$ is reflexive, $E^{*}$ is smooth. Let $f_{x} \rightarrow x$ be the unique support mapping on $E^{*}$. Since $E^{*}$ is smooth, if $\left\{f_{x_{n}}\right\}$ is a sequence in $S\left(E^{*}\right)$ such that $\left\|f_{x_{n}}-f_{x}\right\| \rightarrow 0$, then $f\left(x_{n}-x\right) \rightarrow 0$ for all $f \in S\left(E^{*}\right)$. But the norm and $\sigma\left(E, E^{*}\right)$ topologies agree on $S(E)$, so $\left\|x_{n}-x\right\| \rightarrow 0$; that is, $E^{*}$ is actually $F$-differentiable.

Now let $x=(1,0,0, \ldots)$ and $x_{n}=(0, \ldots, 0,1,0, \ldots)$, where $x_{n}$ has the " $l$ " in the $n$th position. An easy calculation shows that

$$
\|\| x\left\|\left|=1, \quad\left\|\left|x_{n}\right|\right\| \rightarrow 1 \text { and }\left\|\mid x_{n}+x\right\| \| \rightarrow 2 .\right.\right.
$$

But since $x_{n} \rightarrow 0$ in the $\sigma\left(E, E^{*}\right)$ topology, $E$ cannot be WLUR at $x$ in the $f_{x}$ direction for any $f_{x} \in D(x)$.

\section{References}

B. Bollobás (1970), 'An extension to the theorem of Bishop and Phelps', Bull. London Math. Soc. 2, 181-182. 
D. F. Cudia (1963), 'Rotundity', Proc. Amer. Math. Soc. (Convexity) 7, 73-97.

J. Diestel and B. Faires (1974), 'On vector measures', Trans. Amer. Math. Soc. 198, 253-271.

J. Dixmier (1948), 'Sur un théorème de Banach', Duke Math. J. 15, 1057-1071.

J. R. Giles (1971), 'On a characterization of differentiability of the norm of a normed linear space', J. Austral. Math. Soc. 12, 106-114.

J. R. Giles (1975), 'On smoothness of the Banach space embedding', Bull. Austral. Math. Soc. 13, 69-74.

J. R. Giles (1976), 'Uniformly weak differentiability of the norm and condition of Vlasov', $J$. Austral. Math. Soc. Ser. A 21, 393-409.

R. C. James (1964), 'Characterizations of reflexivity', Studia Math. 23, 205-216.

A. Lovaglia (1955), 'Locally uniformly convex Banach spaces', Trans. Amer. Math. Soc. 78, 225-238.

M. A. Smith (1978), 'Some examples concerning rotundity in Banach spaces', Math. Ann. 233, 155-161.

F. Sullivan (1975), 'Some geometric properties of higher duals in Banach spaces', preprint.

A. C. Yorke (1977), 'Weak rotundity in Banach spaces', J. Austral. Math. Soc. Ser. A 24, 224-233.

V. Zizler (1969), 'Some notes on various rotundity and smoothness properties of separable Banach spaces', Comm. Math. Univ. Canol. 10, 2, 195-206.

\section{Mathematics Department}

The University of New England

Armidale, N.S.W. 2351

Australia 